\title{
Investigations on Voltages and Currents in Lightning Protection Schemes Involving Single Tower
}

\author{
Udaya Kumar and Paresh Kumar Nayak
}

\begin{abstract}
A reliable protection against natural lightning has become very essential for modern critical systems. For very vulnerable systems, the protection system is physically isolated from them. In such cases, towers taller than system being protected are generally employed. The maximum allowable bypass current and the so-called rise in potential during the strike decide the height and location of such towers. For tall towers, TM modes dominate at least during the rising portion of the current making the analysis very complicated. In view of this, for a better assessment of the potential at the top and the base currents, experimental investigation in the frequency domain is carried out on the electromagnetically scaled model of the actual tower. The possible reduction in the tower base currents with the connection of ground wires and the influence of neighboring structures on the tower surge response are also studied. Investigations are also carried out on an alternative design involving mast insulated from the supporting tower and a separate set of ground wires acting as down conductors. Some analysis on the electric withstand capabilities of the insulating support is also made. The findings of the present work are believed to be quite useful to lightning protection engineering.
\end{abstract}

Index Terms-Insulated mast design, lightning protection, tower surge impedance, tower with ground wires.

\section{INTRODUCTION}

A RELIABLE protection against lightning has become one of the design criteria for the modern vulnerable systems. For the protection of very critical and sensitive systems, the protection system is generally isolated from the system to be protected. In such cases, towers taller than the system to be protected are generally employed with or without interlinking ground wires.

A reliable design of Lightning Protection Scheme in general demands: 1) an accurate knowledge of the so called rise in potential at various parts; 2) spatio-temporal distribution of the discharge current along the tower and the corresponding electromagnetic fields in the protected volume; and 3) current injected into the soil at the tower base and the consequential rise in soil electric field. An accurate knowledge of the rise in potential basically governs the minimum clearance between the tower and the system being protected. The height of the tower for a given clearance is generally dictated by the allowable current in the bypass/sneaking strokes (shielding failure flashes) [1].

Manuscript received June 2, 2004; revised February 7, 2005. This work was supported in part by a project sponsored by ISRO-IISc Space Technology Cell.

The authors are with the Department of High Voltage Engineering, Indian Institute of Science, Bangalore 560012, India (e-mail: uday@hve.iisc.ernet.in; only2paresh@yahoo.com).

Digital Object Identifier 10.1109/TEMC.2005.853215
The efficacy of the earth termination network associated with the tower is dependent on the peak magnitude as well as the rate of rise of the current injected into it. To ensure safety to the personnel working in the close proximity, it is essential to limit the resulting soil electric gradient to a value lower than a few kilovolts per meter. The soil electric gradient is dependent on the current distribution in it. Another aspect concerning with large electric gradient in soil pertains to sensitive systems housed in buildings that are in the close proximity. In such cases, the resulting soil gradient could interact with the systems through the individual grounds (power, switching, etc.). The peak difference in the potential seen by these local grounds are more or less dominated by the peak of the soil gradient, which in many circumstances, occur at the current peak. In view of these, the peak of the tower base current is considered as a parameter for the study. Furthermore, in a well-designed earthing system, its effective impedance will be much lower than that of the tower and therefore possesses little influence on the current injected into it by the tower.

The present study intends to investigate into the tower top voltage rise as well as the tower base currents during stroke interception by some lightning protection schemes involving single tower. The possible reduction in the tower base currents by the connection of ground wires will be examined. The performance of the insulated mast scheme, wherein, mast is insulated from the supporting tower and a separate set of ground wires act as down conductors, will also be investigated. The intended study will be made by considering a practically existing protection tower. Even though a particular example is considered, it is believed that the various analysis and the topologies studied would give a better insight to the lightning protection engineer. In the author's opinion, such information is rather scarce in the literature.

From the consideration of maximum allowable bypass current, the height for the tower in a particular system is fixed as $130 \mathrm{~m}$, which includes a mast of length $10 \mathrm{~m}$. The protection tower is made of steel and has a base of $16 \times 16 \mathrm{~m}$. Its cross section tapers up to a height of $100 \mathrm{~m}$. From $100 \mathrm{~m}$ onwards, it has uniform cross section of $3 \times 3 \mathrm{~m}$. There is a ladder at the center running up to the full height.

Due to the high rate of rise of the stroke current, at least during the rising portion of the current, higher modes of wave propagation is expected along the protection system. For tall towers, the initial modes are expected to be transverse magnetic (TM), which can later tend to transverse electromagnetic (TEM) and quasi-wave modes. It may be noted here that for the existence of TEM mode on conductors, the net charge/current involved must be zero. In addition, it is preferred that their transverse separation 
distance must be much less than the associated wavelengths. For the case of channel terminated on to the tower, especially for the initial period, these are not strictly satisfied. As a result, the TM mode prevails in the beginning, which makes the analysis for the lightning surge response of protection systems very complicated. Adding on to this, further difficulties arise in the analysis of towers with ground wires and the insulated mast design. Also, it is difficult theoretically to ascertain the possible influence of nearby buildings and other shorter structures.

An important point is to be stressed at this juncture. For the initial lightning surge current, the prevailing mode, as discussed above seems to be TM. It is evident that the definition of the electrostatic potential or the voltage fails for all modes higher than TEM. This is basically due to the significant contribution to the electric field by the time varying magnetic field, which makes the line integral of the electric field vector path dependent. More importantly, the electrical breakdown strength is not well defined for such complex situations. In the absence of any credible alternative, the voltage difference, as defined in the classical sense, will have to be employed for deciding the required clearances. Also, after an initial stage, TEM mode seems to dominate, for which the classical potential difference can be defined.

A number of experimental and theoretical studies have been carried out in the past on the evaluation of the surge impedance of the towers-mostly the electric power transmission line towers. They basically propose some engineering models meant for the evaluation of the rise in potential at the struck point. Models proposed recently are: 1) models composed of many loss less transmission lines [2], [7], [11]; 2) multistory tower models [3]; 3) nonuniform line models [4]; and 4) frequency dependent models [5]. The surge impedance in the above models has been determined either by experiments or by theoretical analysis. Almost all of the above and other reported works were concerned mainly with the rise in tower top potentials for high-voltage power transmission lines. No specific attention is given to the tower base currents. In view of the above, they cannot be adopted for the present study.

Various methods employed in the power engineering literature for the evaluation of the lightning surge response of transmission line towers seems to be relevant to the present study, hence they will be dealt with now.

Theoretical approaches employed in the literature are: 1) TEM mode approximation to the wave propagation on simplified geometries [6], [7]; 2) simplified TM mode on simple conductor arrangements [8]; and 3) computation of the fields using numerical electromagnetic code (NEC-2) [9], [10]. Only the last approach proves to be general and appropriate, however, some difficulties are encountered in the modeling of the interconnected and complicated tower lattice elements. Therefore, although fairly complex tower structures can be simulated, some simplification in the geometry of the actual tower becomes unavoidable. In addition, numerical oscillation problems are encountered when excitation is given to junction formed by short segments. As a result, the validation of the NEC model and the results thus obtained for the protection schemes dealt in the present work is hard to find.
The experimental approaches involved time domain measurements using either time domain reflectometry technique [11] (which also employs a TEM mode approximation to the propagation) or the so called "Volt-Ampere (V-A)"/"Direct method" [12]. In the above, the measurements were made either on the actual towers or on their electromagnetically scaled models. Only the volt-ampere method seems to be more justifiable and is found to give very satisfactory results. Also, studies with scaled models [11], [13], while offering acceptable accuracy, are much more economical and less time consuming. In view of these, the present study intends to adopt the volt-ampere method of experimentation on the scaled models of the system.

\section{PRESENT WORK}

In the earlier methods, the impedance characterization was attempted by resorting to a unit step excitation or to an excitation with fast rising front. However, the response of the tower is obviously dependent on the frequency components of the excitation. At higher frequencies, it behaves more like an antenna, which is followed by transmission line behavior and as the frequency is further reduced a capacitive-inductive and inductive-resistive response can be seen [14]. The frequency spectrum of returnstroke currents extends from dc to tens of megahertz, but the major part of the energy is contained well within $1 \mathrm{MHz}$ or so. Therefore, it is relevant to study the tower behavior for the significant frequency components of the lightning current spectrum rather than to a wide frequency spectrum contained in the step like excitation.

Considering the above, for a better quantification of the system behavior a frequency-domain approach will be employed for the study. Once the behavior of the system is known for a particular range of frequencies, the time domain response for any input could be determined by using Fourier transform techniques, provided the significant components in the frequency spectrum of the input are well within the above range.

\section{A. Experimental Setup}

For the experimental model, a scaling factor close to 1:40 is considered. This was chosen mainly to have some comparison with the published work [9], where a tower of similar height was studied with the above scaling factor. The actual cross sections of the tower elements were rather difficult to reproduce in the model and hence a cylindrical approximation was made according to the geometric mean radii. Further, for the fabrication of the model it was economical to employ standard rod dimensions and use of the same involves one more level of approximation in the model tower. It is evident that such approximation has little influence on the tower response characteristics. The ladder at the center has been approximated by a cylindrical rod. All the intricacies, spanning much less than the wavelengths corresponding to significant frequency components $(<1.5 \mathrm{MHz})$ of the lightning current spectrum have been neglected. The model tower was made up of copper. According to scale model theory [13], the conductivity of the model should be 40 times that of the actual tower. Though, the magnetic permeability of the tower is not scaled exactly, the use of copper for the model 


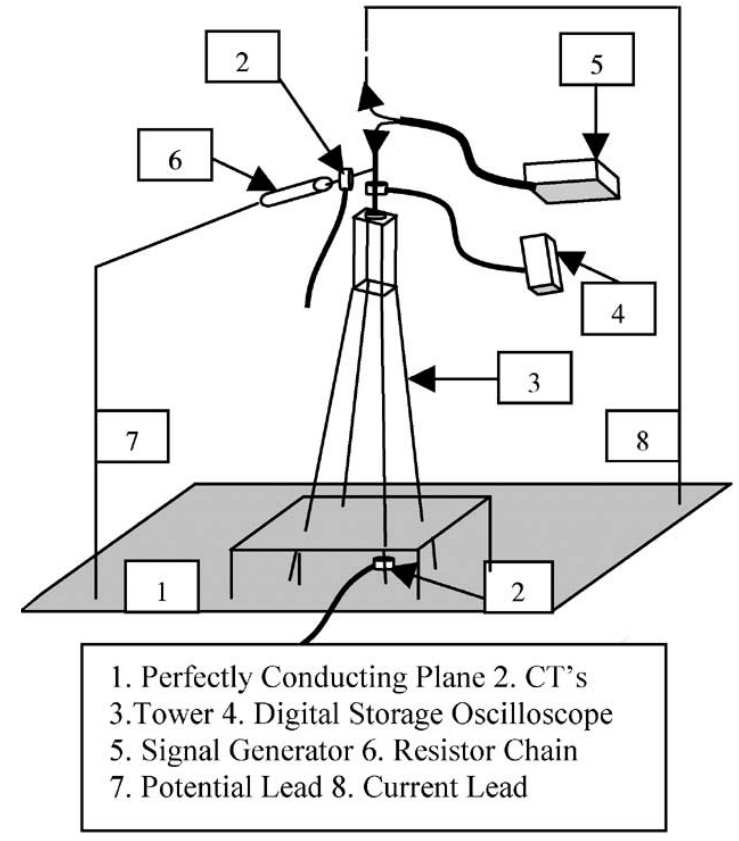

Fig. 1. Schematic of the experimental setup.

attempts to very crudely respect the scale factor for the conductivity. Anyway, these quantities have a very little influence on the response. A representation for the actual tower pile foundation, which extends up to $14 \mathrm{~m}$ in soil, was also attempted by employing rods of uniform cross section. This was purposefully made for a different study, which intends to capture the worst possible response of the grounded portion. Therefore, if the grounded portion is included then the scaling factor correspondingly changes to 35.8 . The mast at the top of the tower was also considered in the scaled model.

The schematic of the experimental setup, made according to the direct method is shown in Fig. 1. The ground plane is approximated by Aluminum plates and foils. In order to incorporate the ground resistance, each of the tower legs is connected to ground through resistances. The net tower footing resistance is $4.8 \Omega$. PVC insulated $7 / 20$ stranded copper wires are used for the current lead and the auxiliary potential lead. According to the literature, a vertical current lead configuration gives the highest value for the "surge impedance" and hence it is chosen for the study. The current lead wire is stretched vertically above the tower top up to a height of about $10 \mathrm{~m}$, after this it extends horizontally to a distance of $7.5 \mathrm{~m}$, and then runs vertically down to the ground. For the tower top voltage measurement, an auxiliary potential wire is stretched perpendicular to the current lead from the tower top and grounded at a point $8.5 \mathrm{~m}$ away from the tower. A wooden ladder supports the instruments as well as the operating person. In the earlier works, a pulse generator is mounted in alignment with the current lead on the top of the tower. This is not only difficult to arrange, but more importantly, due to its physical size, can introduce significant disturbances into the field structure. An attempt has been made in the present work to minimize these disturbances by exciting the system through a coaxial cable $(Z 0=50 \Omega)$ connected to the function generator, which is placed one meter away from the current lead. A series chain of resistance of total value $3.35 \mathrm{k} \Omega$ and length about $60 \mathrm{~cm}$ is inserted between the tower and the potential lead. This value was selected with an intention of keeping it high but at the same time the resulting current should be well within the measurement range of the oscilloscope.

The Pearson make Model 2877 current to voltage converters, with 2-ns rise time, $200 \mathrm{MHz}$ bandwidth and $50 \Omega$ output impedance are used for the noninvasive measurement of various currents. The tower top voltage is calculated by measuring the current flowing through the resistance connected between the tower top and the auxiliary potential lead. These current monitors are connected to the oscilloscope with one-meter long Tektronix probes. The Tektronix Model TH 7104 digital storage oscilloscope employed for the measurements had two independently sampled floating channels with $60 \mathrm{MHz}$ bandwidth, $250 \mathrm{MS} / \mathrm{s}$ sampling rate and 8-bit resolution. However, to avoid possible electromagnetic coupling during simultaneous two channel measurements, only one channel was used at a time. Thirty two times averaging was applied to suppress the effects of noise induced by various broadcasting stations. It may be worth mentioning here that the quantities to be deduced from the experiments are the ratio of two measurements and therefore, the absolute values of the oscilloscope readings are not directly relevant.

Here some more clarifications become necessary. The current measured on the potential lead was dependent on the position of the CT with respect to the resistor. Placing the CT between tower and the resistor yields higher current. For the CT position on middle and the far end of the resistor progressively read lower currents. As a result, the computed time domain voltages for a step like input current, which will be dealt later, was higher than that for CT at the end by about $100 \%$ and $20 \%$, respectively for $\mathrm{CT}$ at the beginning and $\mathrm{CT}$ at the middle. At this juncture, it may be recalled that the placement of resistor on the potential lead is for a convenient replacement of the voltage divider employed in the earlier works [9]. Accordingly, for a measurement corresponding to ground end of the divider, the CT on the potential lead must be placed near the far end. Only with this, a comparison with earlier works becomes more appropriate. In view of this, only for the validation with the earlier works, the measurements corresponding to $\mathrm{CT}$ at the far end of the resistor is utilized. However, due to the small source strength, a more accurate measurement could be made with CT positioned at the beginning. Therefore, when only a comparison of the rise in potential across different configurations is intended, the readings corresponding to $\mathrm{CT}$ at the beginning of the resistor are employed. This aspect has to be recalled in the later sections.

\section{B. Procedure Employed}

Following the earlier works [10], the impedance as seen at the top is defined as the ratio of the top potential to the top current. With regard to the current at the tower base, noting the linearity in the experimental system, the transfer function between the bottom to top current is taken as the ratio of their magnitudes. However, due to the inability of the present experimental set 
up to capture the phase data, only the magnitude data would be obtained. To obtain the relevant time domain quantities phase data will also be required. It is assumed that the travel time delay part can be separated from the phase data. Then only the "minimum phase" data seem to suffice, provided, the travel time could be neglected [15]. For the present work, this travel time is of no serious concern and hence is neglected. Then by using the Hilbert Transform techniques, the required phase data can be extracted from the magnitude spectrum [15], [16]. This approach has been adopted for all time domain calculations dealt in the present work.

\section{Investigation Results}

As the waveform of the lightning current is not a fixed entity, time domain data for representative cases will be evaluated. For this, strokes with front times close to $1.1,1.8,3.5$, and $5.5 \mu \mathrm{s}$ are selected. These front times are exceeded respectively by $50 \%$ of negative subsequent strokes, $95 \%$ of the negative first strokes, $95 \%$ of the positive flashes and $50 \%$ of negative first strokes [17]. Incidentally, for most of the intended time domain calculations, results up to $40 \mathrm{MHz}$ are found to be adequate. As both the impedance at the top as well as the base currents are linear function of the input stroke current, the time domain calculations are carried out with a current of unit amplitude. This will aid in the evaluation of the actual magnitudes as well as the normalized comparison across different wave fronts. From the lightning current measured on instrumented towers (both short as well as tall), many functional forms were suggested [17] and amongst them the double exponential forms is chosen for the intended time domain calculations. It is worth noting here that the two quantities of interest attain their peak around or within the time to peak of the stroke current and, therefore, the details of the tail region of the stroke current is not that important.

The experimentally deduced impedance and current transfer functions for vertical conductor configuration, differed from that obtained through NEC. However, only a simplified model was simulated in NEC and the experimental conditions were not very ideal. The over all accuracy of the results, which is dependent on the accuracy of measurement, adequacy of the range of frequencies used in the experiments and the effectiveness of the experimental ground plane, is believed to be within 10\%-15\%. The simulation for the vertical conductor configuration in NEC, as well as some extensive simulation with SPICE for transmission lines, indicate that the present method of evaluating the phase data did not show any appreciable error for a time period of $10 \mu$ s or so.

For the validation of the present work, impedance of the single vertical conductor was computed from the measured frequency domain impedance function. Instead of a step excitation with $0.2 \mu$ s time to peak used in [9], the present work employs a time to peak of $0.4 \mu \mathrm{s}$. This was necessary to make the higher significant frequency components to be within the range of frequencies of the experimental data. Using the procedure employed in [9], the value of the computed impedance is $375 \Omega$. The value quoted in [9] is about $360 \Omega$, however, it was for a horizontal current injection. For a vertical current injection, it is supposed to be

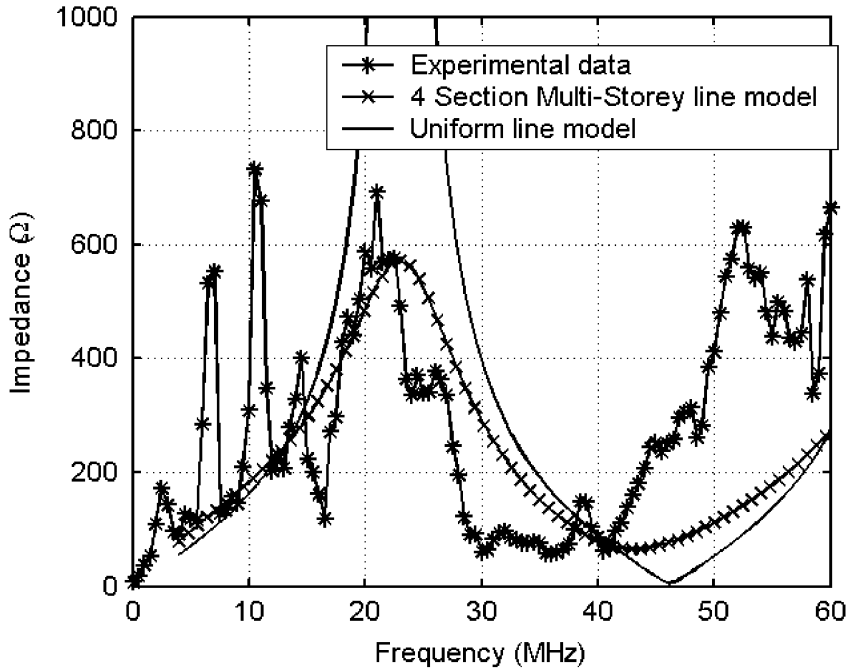

(a)

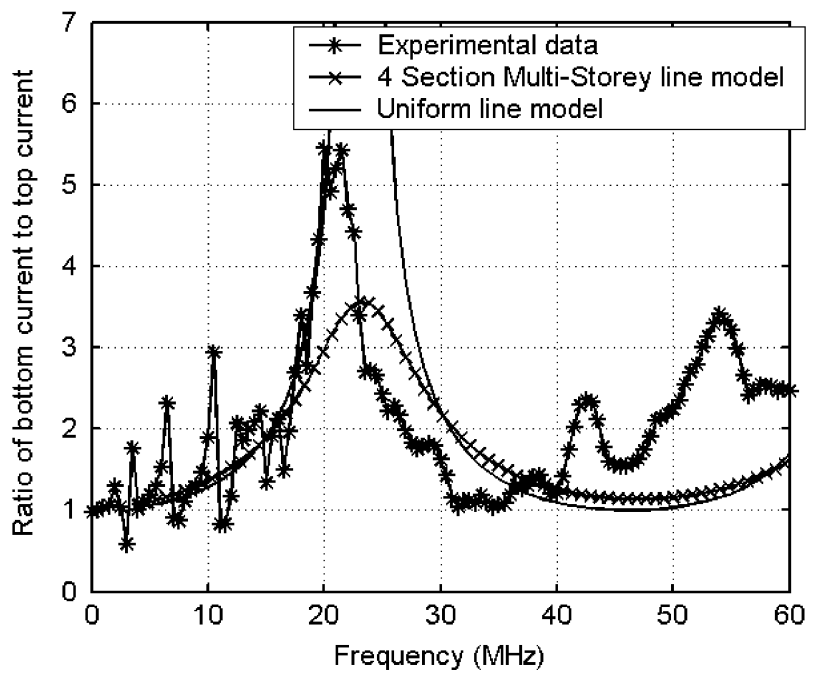

(b)

Fig. 2. Experimentally obtained impedance and base current characteristics for 1:40 scaled model: (a) Magnitude of the impedance function and (b) magnitude of the current transfer function.

about $10 \%$ higher, thus making it to be around $396 \Omega$. Therefore, the predicted value is quite accurate, thus proving the validity of the present work. As mentioned earlier, this value corresponds to $\mathrm{CT}$ positioned at the far end of the resistor in the potential lead.

Further, for many of the configurations dealt below, NEC simulations could be and have been carried out with very simplified tower geometry. Here the tower is represented by its four main legs and only interconnections at the top. The various time domain values computed from the simulation results are found to be supportive of the investigation results. This provides a further validity to the present work.

1) Isolated Tower: The impedance and the current transfer functions obtained from the measured frequency domain data on isolated tower model are shown in Fig. 2. From the figures, it is obvious that apart from the initial slender peaks, wide dominant peaks are seen in both impedance as well as the current 
transfer functions around 21 and $54 \mathrm{MHz}$ respectively. These indicate very dispersive surge propagation on the tower. From the NEC-2 runs for single vertical conductor, it was verified that the initial slender peaks are due to the interaction with current lead wire. For a comparison, the impedance and the current transfer function for the uniform line (i.e., line with constant surge impedance) and the multi-story line models to transmission line towers are also shown on the same figure. It is evident from the figures that the characteristic of the tower is distinctly different from that of uniform transmission line approximation. However, the comparison with the multistory tower model seems to be relatively better.

For the comparison with the reported data [9], the impedance of the tower under study has been computed using the earlier said procedure. The computed tower top impedance accordingly is about $190 \Omega$, quite higher than the value of $150 \Omega$ given in [9]. This difference can be mainly attributed to the difference in the cross section of the towers. The base and top dimensions of the tower studied in [9] are 22 and $10 \mathrm{~m}$, respectively, which are relatively quite high. In addition, in the present case, there is a 10-m long mast at the top. In view of these, the above difference in the impedance could be justified.

Along the current lead wire, which is an attempt to simulate the stroke channel, the velocity of propagation is close to that of light $(c)$. Keeping this in mind, in order to ascertain the dependency of the impedance and current transfer functions on the reduced velocity of propagation of the stroke currents, NEC runs were made with a loaded channel terminated on to a vertical conductor. Appreciable differences were not found even for the current propagation velocity of $c / 3$. This shows the adequacy of the channel representation made in the present experimental setup.

The computed voltages and currents for representative stroke currents of unit amplitude are as shown in Fig. 3. The tower response can be seen to be of highly damped nature and quite different from that of the uniform transmission lines. The peak values of the top voltage and the bottom currents computed for stroke currents with different time to front are summarized in Table I.

2) Tower With Ground Wires: In many situations, it is desired for various reasons that, during a strike, the rise in tower top potential must be reduced for an existing tower. Also, the stroke current entering the soil produces large electrical gradients and a rise in local potential. As these can be dangerous to both the personnel and the critical system situated in the close proximity, it is desired that a portion of the current must be diverted away. The simplest and possibly the only solution seem to be the erection of ground wires connecting towers to remote earth. Such an effort has been suggested for reducing surge impedance of the electrical power transmission line towers [18]. However, there seems to be a little work (including the present one), which can be generalized.

In the present work, to ascertain the effectiveness of the ground wires, some experiments are conducted and only the results, which are relevant, will be presented. The parameters chosen for the study are the number of ground wires, which are varied from one to four, location of their connection to the tower

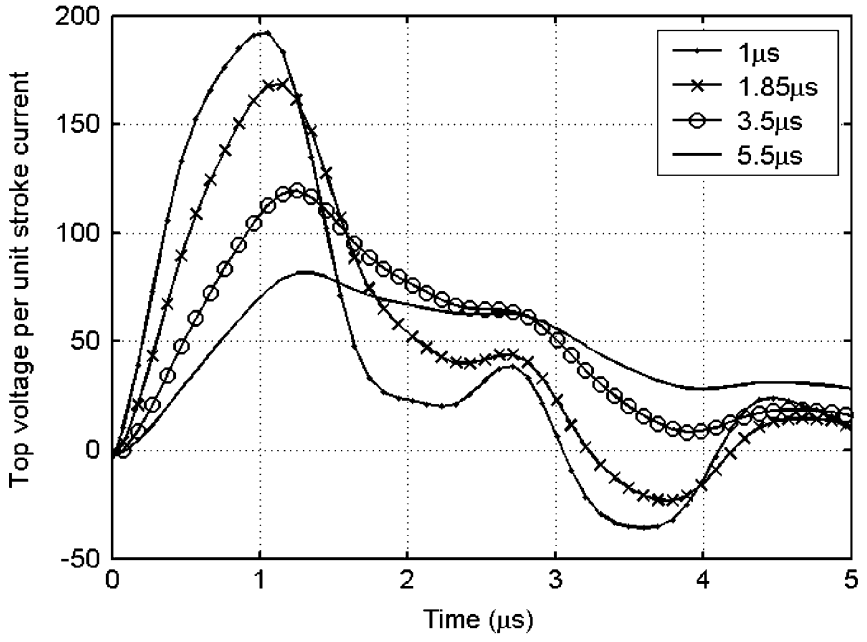

(a)

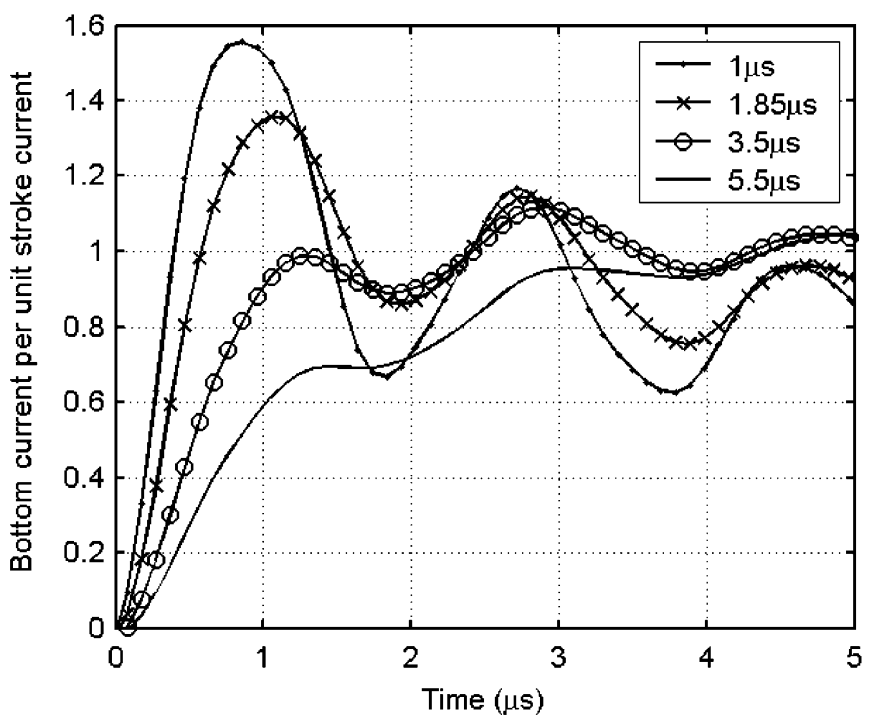

(b)

Fig. 3. Time domain results for the protection tower: (a) Computed top voltages and (b) computed base currents.

TABLE I

TOP Voltages AND BASE CURRENTS FOR UNIT STROKE CURRENT

\begin{tabular}{c|c|c}
\hline Time $(\mu \mathrm{s})$ & Top voltage $(\mathrm{V})$ & Base current $(\mathrm{A})$ \\
\hline 1.00 & 191.8 & 1.56 \\
\hline 1.85 & 168.2 & 1.36 \\
\hline 3.50 & 119.5 & 1.12 \\
\hline 5.50 & 81.6 & 1.04 \\
\hline
\end{tabular}

which were at $H, 0.75 H$, and $0.5 H$ (where " $H$ " is the height of the tower, excluding the mast) and the grounding locations, which are chosen at $5.66 \mathrm{~m}(\mathrm{P} 1), 2.83 \mathrm{~m}(\mathrm{P} 2)$, and $1.55 \mathrm{~m}(\mathrm{P} 3)$ away from the center of the tower. These correspond to the actual distances of, respectively, $202 \mathrm{~m}, 101 \mathrm{~m}$, and $55.5 \mathrm{~m}$. To minimize the coupling from the ground wire to the potential lead, their location is always selected opposite to the potential lead. Copper wires of 1-mm diameter are employed as ground wires in the experimentation. 
At the beginning, experiments are conducted by connecting single ground wire to the tower according to the above-described connection. It was found that the reductions in the impedance as well as the tower base current were not very significant. Therefore, it was decided to employ more number of ground wires. However, for the two ground wire case, earthing at P1 and $\mathrm{P} 2$ with ground wire connections to tower at the top only was chosen. This is because in the case of single ground wire it was observed that the wire earthed very close to the tower had very little influence on the impedance and current transfer functions. For ascertaining the effect of coupling between the ground wires, experiments are conducted for two different separation distances $(D)$. These distances are chosen to be 2.5 and $0.5 \mathrm{~m}(58 \%$ and $12 \%$ of their length respectively) at P2 and 4.5 and $1.5 \mathrm{~m}(68 \%$ and $23 \%$ of their length, respectively) at P1. The impedance functions as well as the current transfer functions obtained clearly show that the connections of two ground wires cause an overall reduction in the magnitude of both the functions. However, the differences in both the functions corresponding to the two separation distances are not very significant.

For a possible further reduction in both the quantities, four ground wires with two different earthing distances are also employed. Two ground wires were earthed at P1 and other two at $\mathrm{P} 2$. Further, it was felt that fixing all the four ground wires at the tower top can lead to practical difficulties and experiments were also conducted with two ground wires connected to the tower at a height of $H$ and the rest two were connected to the tower at a height of $0.75 H$. The corresponding impedance functions as well as the current transfer functions, as compared to the twoground wire configurations, clearly show an appreciable overall reduction. The magnitude of the current transfer function shows much more appreciable reduction.

The reduction in the computed peak amplitudes of the top voltages and the ground end currents for the above-discussed configurations are summarized in Table II. The computed base currents for the fastest representative stroke current are given in Fig. 4. The above table shows that even though there is an overall reduction in both the quantities, occasional increase in the peak of the top voltages, where the increase is within the accuracy of the measurement, can also be observed. The existence of an intermittent peak in the frequency domain impedance function of the tower with ground wires can be responsible for this opposite trend. As the magnitude of this intermittent peak is relatively smaller, so is the increase in the computed time domain top voltages. Fig. 4 clearly shows that the base current for tower with ground wires differs significantly from that for the isolated tower, especially after the first peak.

3) Tower With Neighboring Conducting Objects: In many practical situations, smaller towers, reinforced concrete buildings, etc., are generally present in the close proximity of the tower. In such cases, two issues become important. The first one is the influence of such structures on the response of the protection tower and the second issue is the magnitude of the induction to them. The present work limits itself to ascertain only the first. As the number of cases and parameters involved are quite large, only the worst-case scenarios are considered.
TABLE II

REDUCTION IN TOWER TOP VOLTAGES AND BASE CURRENTS WITH GROUND Wires. (a) PERCENTAge Reductions in TOWER TOP Voltage. (b) Percentage Reductions in TOWER Base CuRrent

\begin{tabular}{c|c|c|c|c|c}
\hline \multirow{2}{*}{$\begin{array}{c}\text { No. } \\
\text { of } \\
\text { GW }\end{array}$} & \multirow{2}{*}{$\begin{array}{c}\text { Different } \\
\text { arrangements }\end{array}$} & \multicolumn{4}{|c}{$\begin{array}{c}\text { Reduction in top voltages with } \\
\text { respect to isolated tower }\end{array}$} \\
\cline { 2 - 6 } & & $1 \mu \mathrm{s}$ & $1.85 \mu \mathrm{s}$ & $3.5 \mu \mathrm{s}$ & $5.5 \mu \mathrm{s}$ \\
\hline \multirow{4}{*}{1} & $\mathrm{P} 1, \mathrm{H}$ & 11.2 & 2.0 & 19.5 & 19.6 \\
& $\mathrm{P} 1,0.75 \mathrm{H}$ & 0.50 & 12.0 & 14.8 & 15.4 \\
\cline { 2 - 6 } & $\mathrm{P} 2, \mathrm{H}$ & 9.0 & 10.5 & 17.0 & 15.7 \\
& $\mathrm{P} 2,0.75 \mathrm{H}$ & -2.6 & -1.8 & 9.4 & 9.0 \\
& $\mathrm{P} 2,0.5 \mathrm{H}$ & -1.5 & -1.5 & 13.5 & 14.3 \\
\cline { 2 - 6 } & $\mathrm{P} 3, \mathrm{H}$ & 8.4 & 9.2 & 17.0 & 17.5 \\
& $\mathrm{P} 3,0.75 \mathrm{H}$ & -6.6 & -6.0 & 6.5 & 6.9 \\
& $\mathrm{P} 3,0.5 \mathrm{H}$ & -1.8 & -1.4 & 8.5 & 9.4 \\
\hline \multirow{4}{*}{2} & $\mathrm{P} 1, \mathrm{H}$ & 16.5 & 17.0 & 26.5 & 26.2 \\
\cline { 2 - 6 } & $\mathrm{P} 2, \mathrm{H}$ & 16.0 & 18.5 & 27.0 & 26.0 \\
\hline \multirow{4}{*}{4} & $\mathrm{P} 1, \mathrm{P} 2, \mathrm{H}$ & 16.2 & 17.7 & 26.6 & 28.0 \\
\cline { 2 - 6 } & $\mathrm{P} 1, \mathrm{H}, \mathrm{P} 2,0.75 \mathrm{H}$ & 13.2 & 13.9 & 26.4 & 25.0 \\
\hline
\end{tabular}

(a)

\begin{tabular}{c|c|c|c|c|c}
\hline \multirow{2}{*}{$\begin{array}{c}\text { No. } \\
\text { of } \\
\text { GW }\end{array}$} & $\begin{array}{c}\text { Different } \\
\text { arrangements }\end{array}$ & \multicolumn{4}{|c}{$\begin{array}{c}\text { \% Reductions in the base currents with } \\
\text { respect to isolated tower }\end{array}$} \\
\cline { 2 - 6 } & & $1 \mu \mathrm{s}$ & $1.85 \mu \mathrm{s}$ & $3.5 \mu \mathrm{s}$ & $5.5 \mu \mathrm{s}$ \\
\hline \multirow{4}{*}{1} & $\mathrm{P} 1, \mathrm{H}$ & 16.8 & 10.0 & 16.5 & 14.3 \\
& $\mathrm{P} 1,0.75 \mathrm{H}$ & 12.8 & 7.1 & 16.4 & 11.0 \\
\cline { 2 - 6 } & $\mathrm{P} 2, \mathrm{H}$ & 21.2 & 16.4 & 24.0 & 22.8 \\
& $\mathrm{P} 2,0.75 \mathrm{H}$ & 17.4 & 12.2 & 18.2 & 16.2 \\
& $\mathrm{P} 2,0.5 \mathrm{H}$ & 10.2 & 11.9 & 18.6 & 21.0 \\
\cline { 2 - 6 } & $\mathrm{P} 3, \mathrm{H}$ & 19.8 & 17.0 & 23.8 & 23.2 \\
& $\mathrm{P} 3,0.75 \mathrm{H}$ & 17.1 & 14.6 & 19.9 & 19.5 \\
& $\mathrm{P} 3,0.5 \mathrm{H}$ & 15.0 & 14.1 & 21.2 & 23.2 \\
\hline \multirow{4}{*}{2} & $\mathrm{P} 1, \mathrm{H}$ & 23.3 & 18.2 & 23.4 & 26.7 \\
\cline { 2 - 6 } & $\mathrm{P} 2, \mathrm{H}$ & 28.2 & 27.9 & 34.5 & 35.7 \\
\hline \multirow{4}{*}{4} & $\mathrm{P} 1, \mathrm{P} 2, \mathrm{H}$ & 40.1 & 36.0 & 41.8 & 42.2 \\
\cline { 2 - 6 } & $\mathrm{P} 1, \mathrm{H}, \mathrm{P} 2,0.75 \mathrm{H}$ & 40.0 & 35.8 & 38.5 & 41.2 \\
\hline
\end{tabular}

(b)

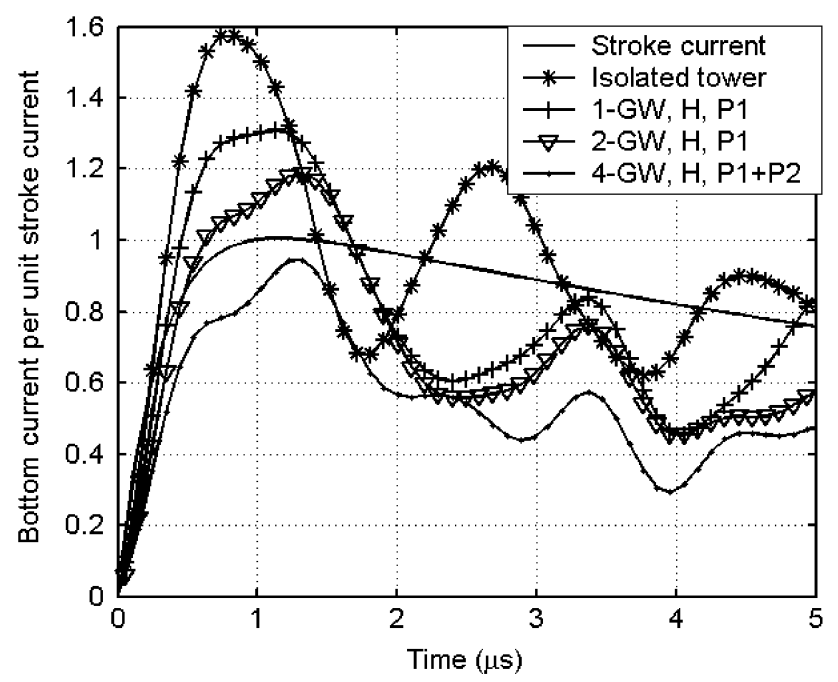

Fig. 4. Computed base currents for tower with ground wires (GW).

Accordingly, it is presumed that a grounded conducting surface parallel to the tower's side face would emulate the required situation. Experimental representation for the same is made by placing Aluminum sheet of $60 \mathrm{~cm}$ width and $0.1 \mathrm{~mm}$ thickness (which corresponds to $24 \mathrm{~m}$ width and $4 \mathrm{~cm}$ thickness in 


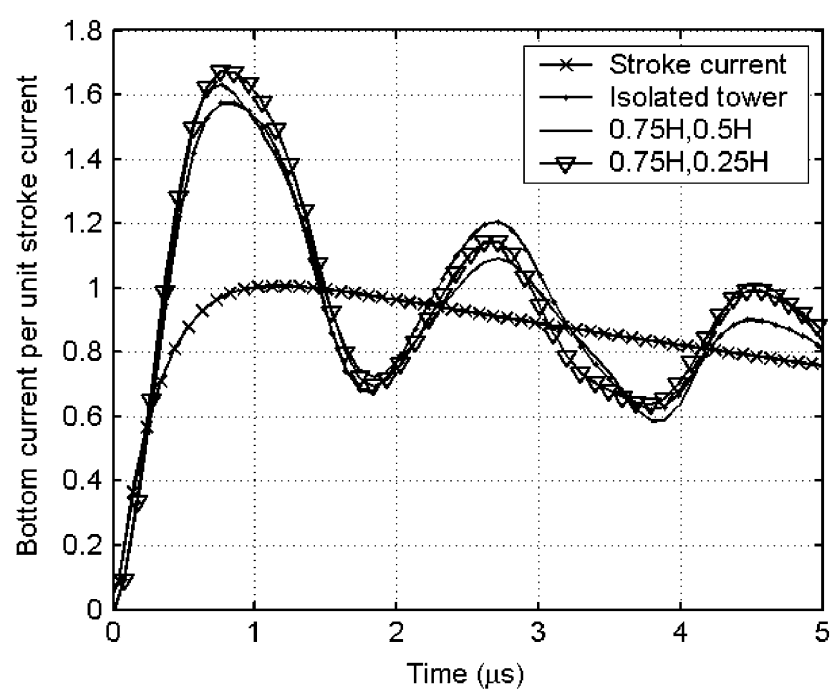

Fig. 5. Tower base currents for different height and placement of grounded conducting surface.

the actual scale). The influence of the actual structure can be anticipated to be much lower than the above.

Experiments are conducted by erecting the sheet at two different locations, 0.25 and $0.5 \mathrm{H}$ away from the tower center. The height for the sheet was set to 0.75 and $0.5 \mathrm{H}$ at the first location and $0.75 \mathrm{H}$ at the second location. For the quantitative analysis, time domain voltages and the base currents are computed. These results show that only for sheet of height $0.75 \mathrm{H}$ placed $0.25 \mathrm{H}$ away from the tower, some influence could be seen on the peak values (refer to Fig. 5). In view of the this and noting that the representation made was an attempt to simulate the worst possible situations, it can be concluded that in most of the practical situations, the presence of neighboring structures/buildings (which invariably are much shorter than the tower), have little influence on the peak tower top voltages and tower base currents.

4) Tower With Insulated Mast and Separate Down Conductors: In the protection scheme for very sensitive and susceptible systems, it is highly desirable to convey the current in the intercepted stroke to remote earth, away from the protected system. This would minimize the secondary effects such as rise in earth potential and the electromagnetic induction. In an attempt to realize this, some of the practical lightning protection schemes insulate the mast from the tower and employ ground wires from the masts to remote earth. The success of such a scheme mainly depends on the dielectric withstand capabilities of the insulator support. This is in turn dependent apart from other issues, on the rise in tower top potential. In order to assess the rise in potential, few experiments are conducted. The length considered for the insulating cylinder is $10 \mathrm{~m}$. The parameters for the study are the number of ground wires (varied from one to four), the separation distance $(D)$ between the wires and their earthing distances from the supporting tower.

The impedance functions obtained from the experimentation on different configurations indicate that except for the case of four ground wires, the magnitude of the impedance functions, as compared to that of an isolated tower, are quite higher. Further,
TABLE III

COMPARISON OF VARIOUS PARAMETERS FOR InSUlATED MAST DESIGN. (a) PERCENTAGE INCREASE IN THE TOP Voltages. (b) COMPUTED GROUND WiRE CURRENTS AT THE BASE AND INDUCED TOWER BASE CURRENTS

\begin{tabular}{c|c|c|c|c|c}
\hline \multirow{2}{*}{$\begin{array}{c}\text { No. of } \\
\text { ground } \\
\text { wires }\end{array}$} & \multirow{2}{*}{ Arrangement } & \multicolumn{4}{|c}{$\begin{array}{c}\text { \% Increase in top voltages with respect } \\
\text { to that for the supporting tower }\end{array}$} \\
\cline { 3 - 6 } & & $1 \mu \mathrm{s}$ & $1.85 \mu \mathrm{s}$ & $3.5 \mu \mathrm{s}$ & $5.5 \mu \mathrm{s}$ \\
\hline \multirow{3}{*}{1} & $\mathrm{P} 1$ & 91.4 & 104.4 & 117.5 & 141.8 \\
& $\mathrm{P} 2$ & 101.2 & 125.9 & 137.8 & 141.0 \\
& $\mathrm{P} 3$ & 95.0 & 123.9 & 123.2 & 123.2 \\
\hline \multirow{4}{*}{2} & $\mathrm{P} 1, \mathrm{D}=4.5 \mathrm{~m}$ & 32.6 & 43.5 & 58.0 & 73.8 \\
& $\mathrm{P} 1, \mathrm{D}=0.08 \mathrm{~m}$ & 49.5 & 64.9 & 79.8 & 98.2 \\
\cline { 2 - 6 } & $\mathrm{P} 2, \mathrm{D}=1.5 \mathrm{~m}$ & 45.5 & 115.6 & 59.2 & 61.2 \\
\hline \multirow{2}{*}{4} & $\mathrm{P} 1, \mathrm{P} 2, \mathrm{H}$ & 3.5 & 8.0 & 14.4 & 16.2 \\
\hline
\end{tabular}

(a)

\begin{tabular}{|c|c|c|c|c|c|}
\hline \multirow{2}{*}{$\begin{array}{l}\text { Arrang- } \\
\text { ement }\end{array}$} & \multirow{2}{*}{$\begin{array}{l}\text { Different } \\
\text { currents }\end{array}$} & \multicolumn{4}{|c|}{$\begin{array}{l}\text { Ratio of the peak base current to } \\
\text { peak of the input current }\end{array}$} \\
\hline & & $1 \mu \mathrm{s}$ & $1.85 \mu \mathrm{s}$ & $3.5 \mu \mathrm{s}$ & $5.5 \mu \mathrm{s}$ \\
\hline \multirow{2}{*}{$\begin{array}{l}\text { 1-GW, } \\
\text { P1 }\end{array}$} & I-ground end & 1.84 & 1.83 & 1.58 & 1.24 \\
\hline & I-tower-base & 0.44 & 0.36 & 0.24 & 0.15 \\
\hline \multirow{2}{*}{$\begin{array}{l}\text { 2-GW, } \\
\text { P1 }\end{array}$} & I-ground end & 1.84 & 1.81 & 1.58 & 1.23 \\
\hline & I-tower-base & 0.47 & 0.37 & 0.24 & 0.16 \\
\hline \multirow{2}{*}{$\begin{array}{l}\text { 1-GW, } \\
\text { P2 }\end{array}$} & I-ground end & 1.90 & 1.75 & 1.36 & 1.15 \\
\hline & I-tower-base & 0.60 & 0.50 & 0.34 & 0.30 \\
\hline \multirow{2}{*}{$\begin{array}{l}\text { 2-GW, } \\
\text { P2 }\end{array}$} & I-ground end & 1.83 & 1.69 & 1.30 & 1.20 \\
\hline & I-tower-base & 0.62 & 0.51 & 0.36 & 0.25 \\
\hline \multirow{2}{*}{$\begin{array}{l}\text { 1-GW, } \\
\text { P3 }\end{array}$} & I-ground end & 1.86 & 1.68 & 1.27 & 1.15 \\
\hline & I-tower-base & 0.66 & 0.55 & 0.37 & 0.25 \\
\hline 4-GW & I-ground end & 1.72 & 1.61 & 1.27 & 1.12 \\
\hline $\mathrm{P} 1, \mathrm{P} 2$ & I-tower-base & 0.52 & 0.46 & 0.32 & 0.22 \\
\hline
\end{tabular}

(b)

the numbers of peaks are more, which implies that the benign negative reflections arising at the ground end now needs more time to reach the top. Therefore, the potential rise for this scheme can be expected to be higher.

Another important issue, which is envisaged in the present work for this scheme is the possible induction to the tower. For the quantification of the same, the tower bottom currents were measured. These measurements prove that some significant induction occurs to the tower. For the present configuration, this induction seems to be dominant below $750 \mathrm{kHz}$ (which corresponds to about $30 \mathrm{MHz}$ in the scaled model) and the peaking occurs at the peaking of the ground end current in the ground wires. It is experimentally verified that this induced current is not circulating within the tower legs and hence must be forming some sort of standing waves along the tower.

The peak magnitudes of the computed time domain mast voltages and the induced tower base currents for the configurations studied in this work are summarized in Table III. Some sample results are shown in Fig. 6. From the Table III, it is clear that for all cases with single- and double-ground wires, the magnitude of the tower top voltages are significantly higher than that for 


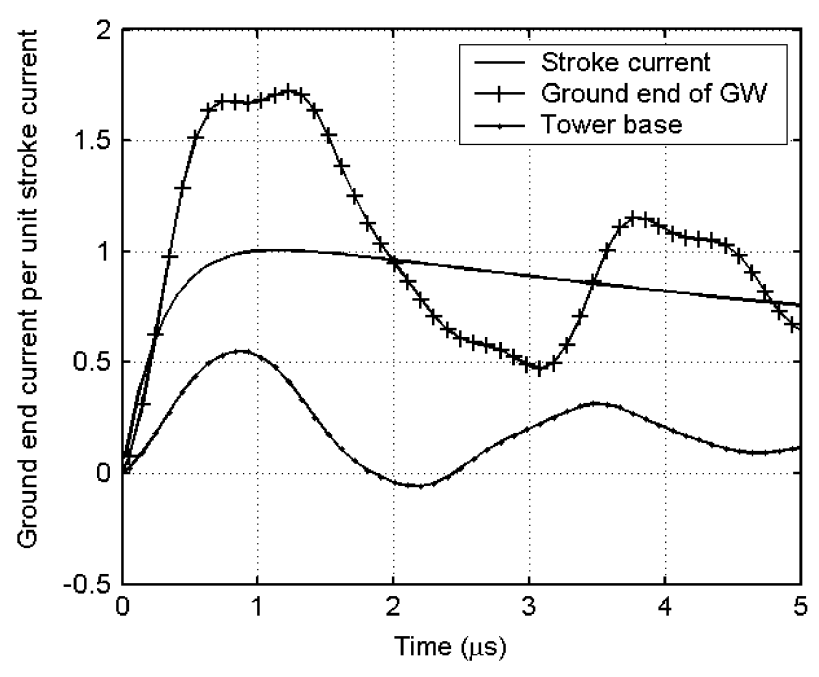

Fig. 6. Computed ground end currents for tower with insulated mast.

an isolated tower. Only for the four-ground wire configuration, the top voltages are in better comparison with that of an isolated tower at least for the faster rise times. Table III(b), shows that the induction to the tower becomes very significant for faster front currents and the peak value of the induced base current can reach even $50 \%$ of the incident stroke current. With regard to these induced tower currents measured at the base, the influence of the ground wire arrangements is not very evident. This finding is in contrast to the original idea of diverting all the current away from the protected system. However, if this scheme were to be successful, then as compared to the conventional schemes, the tower base currents will be smaller in their magnitudes and higher in their frequency content.

Apart from the above, the presumed success of the insulating mast scheme, especially for strokes with large current magnitudes, is solely dependent on the electrical withstand capabilities of the insulating support. It is well known in insulation engineering that once a surface is introduced, the net electrical strength can go below that of the original air gap itself. In other words, the bulk breakdown strength of the insulating support will not be of any concern at all. Instead, it is the surface breakdown strength along the insulator surface that needs to be considered. This surface strength, similar to the electrical power transmission line power frequency pollution flashover problems, could be affected by the natural/artificial pollution. No credible data seems to exist for impulse flashover strength (whether dry or wet) of long surfaces of length exceeding 10-15 $\mathrm{m}$ and more so under polluted conditions. Depending on the type of the insulator, it may also become necessary to consider the effect of space charge accumulation around the insulator surface. Another point to be attended to here is the following. For the required clearance, which could be estimated, to be in the order of tens of meters, insulating supports may have to be assembled vertically. In such situations, the metallic joints can prove to be detrimental. Under various circumstances, they can initiate bidirectional streamers, which can culminate into a full flashover.

In other words, the prevailing significant induction to the tower and the actual breakdown strength along the support insulator seem to be disfavoring the original philosophy of the insulated mast scheme.

\section{Further Analysis}

For providing additional scrutiny, NEC simulations were carried out on simple vertical conductor of same height. Mainly two issues are addressed. The first one pertained to the location of the maximum rate of rise on the current front and the performance of the tower for faster subsequent strokes. In the measured lightning current waveforms, the maximum rate of rise for subsequent strokes is reported to be located on the upper half of the wave front rather than at the origin (which is enforced in the double exponential form employed for all the preceding calculations). In order to ascertain the effect of the same, using the NEC simulations results on vertical conductor, time domain quantities were computed for 1) double exponential current with $1.1 \mu$ s time to crest and 2) current waveform with Gaussian front: $\exp \left(-\left(\left(t-t_{0}\right)^{2} / \mu\right)\right)$, which is followed by an exponential decay. The peak amplitude and the maximum rate of rise of the current in the latter were made same as that of the former. The computed top potentials and bottom currents for the above two cases were well within 5\%-6\%. This shows that for a given maximum rate of rise of the current, the actual location of the fast rising portion on the wave front is not very important. The next point is with regard to the very fast subsequent return strokes, for which the range of experimentally deduced frequency domain functions will be stretched to their limits. In order to ascertain this, by employing a step like excitation with linear front, the peak amplitudes of the top potentials and bottom currents were computed for the vertical conductor geometry. When the time to crests is varied between $(0.2-0.6 \mu \mathrm{s})$, the maximum differences in the computed values were well within $6 \%$. This shows that so long as the time to crest is held lower than the two-way travel time along the down conductor, the actual time to crest possesses insignificant influence.

The second issue dealt was on the adequacy of the predicted tower top potentials under the condition that the neighboring structure could have significant induction. It has already been shown in an earlier section that the influence of such structures on the tower top potentials and the bottom currents are not very significant. Therefore, these quantities can be quoted without explicitly referring to the structure under protection. The following simulation results will provide further justifications. Simulations were carried out on thin vertical conductors with radius, height and the separation distance of the protected conductor as the study variables. By computing the current through a resistor chain connected along the shortest path between the conductors, the so-called potential difference defined as the path integral of the electric field, was determined. The results show that the peak amplitude of the potential difference remains the same even when the height of the protected conductor is only $50 \% H$ (where $H$ is height of the protecting conductor) and further for the range of separation distance which was varied between $10 \%-30 \% H$, the potential difference showed a marginal difference of only $20 \%$. All these facts strongly support the 
argument that the tower quantities can be evaluated without referring to the system under protection.

\section{CONCLUSION}

The present work has investigated into the rise in potential and the ground end currents during stroke interception by the protection schemes involving single tower. Experimental approach using the electromagnetically scaled model was employed for the study. Even though studies were made for a practical tower of $130 \mathrm{~m}$ height, the configurations studied and the corresponding results, are believed to be quite useful to lightning protection engineering in general. A frequency domain approach has been employed for the better characterization of the protection system. Many of the results obtained in the present investigations are believed to be quite novel. The results obtained can be summarized as follows.

- Both the tower top potential as well as the tower base current exhibits a damped sort of time domain response indicating a dispersive propagation along the tower. Especially for faster and medium front time currents, this will result in significant deviation from the commonly employed uniform line approximation.

- By connecting multiple ground wires to the tower, it is possible to reduce both the tower base currents and the tower top voltages. However, the reduction in current is higher than the reduction in the top voltage.

- The experimental study for the tower with nearby conducting structures show that in most of the practical situations, the presence of neighboring structures/buildings, (which invariably are shorter than the tower) have insignificant influence on the peak amplitude of the top voltages as well as the base currents.

- The studies made on the insulated mast scheme, which is an alternative design philosophy, show that the rise in top potential is always higher. A comparable rise in top potential could be achieved only by connecting multiple ground wires (four or more). There would be significant induced currents in the tower base, whose magnitude increases with increase in steepness of the stroke current. Some relevant points on the insulator surface flashover were also presented.

\section{REFERENCES}

[1] U. Kumar and N. T. Joseph, "Analysis of air termination system of the lightning protection scheme for the Indian satellite launch pad," Proc. Inst. Elec. Eng., Sci. Meas. Technol., vol. 150, no. 1, pp. 3-10, Jan. 2003.

[2] T. Hara and O. Yamamoto, "Modelling of a transmission tower for lightning surge analysis," Proc. IEE Gener. Transm. Distr., vol. 143, no. 3, pp. 283-289, May 1996.

[3] T. Yamada, A. Mochizuki, E. Zaima, A. Ametani, M. Ishii, and S. Kato, "Experimental evaluation of a UHV tower model for lightning surge analysis," IEEE Trans. Power Del., vol. 10, no. 1, pp. 393-402, Jan. 1995.
[4] J. A. Gutiérrez, R. P. Moreno, J. L. Naredo, J. L. Bermúdez, M. Paolone, C. A. Nucci, and F. Rachidi, "Nonuniform transmission tower model for lightning transient studies," IEEE Trans. Power Del., vol. 19, no. 2, pp. 490-496, Apr. 2004.

[5] A. Ametani, Y. Kasai, J. Sawada, A. Mochizuki, and T. Yamada, "Frequency-dependent impedance of vertical conductors and a multiconductor tower model," Proc. IEE Gener. Transm. Distr., vol. 141, pp. 339345, Jul. 1994.

[6] C. F. Wagner and A. R. Hileman, "A new approach to the calculation of the lightning performance of transmission lines III-A simplified method: Stroke to tower," AIEE Trans. Power App. Syst., vol. 79, pp. 589-603, Oct. 1960.

[7] M. A. Sargent and M. Dervenzia, “Tower surge impedance," IEEE Trans. Power App. Syst., vol. 88, no. 5, pp. 680-687, May 1969.

[8] H. Motoyama and H. Matsubara, "Analytical and experimental study on surge response of transmission line tower," IEEE Trans. Power Del., vol. 15, no. 2, pp. 812-819, 2000.

[9] M. Ishii and Y. Baba, "Numerical electromagnetic field analysis of tower surge response," IEEE Trans. Power Del., vol. 12, no. 1, pp. 916-921, Jan. 1997.

[10] Y. Baba and M. Ishii, "Numerical electromagnetic field analysis on measuring methods of tower surge response," IEEE Trans. Power Del., vol. 14, no. 2, pp. 630-635, Apr. 1999.

[11] W. A. Chisholm, Y. L. Chow, and K. D. Srivastava, "Lightning surge response of transmission towers," IEEE Trans. Power App. Syst., vol. 102, no. 9, pp. 3232-3242, Sep. 1983.

[12] M. Kawai, "Studies of the surge response of a transmission line tower," IEEE Trans. Power App. Syst., vol. 83, pp. 30-34, Jan. 1964.

[13] J. G. Anderson and J. H. Hagenguth, "Magnetic fields around a transmission line tower," AIEE Trans. Power App. Syst., vol. 78, pp. 1391-1398, Oct. 1959.

[14] T. Gupta "High frequency response of tall towers," M.E. Thesis, Dep. High Voltage Eng., Indian Inst. Sci., Bangalore, India, Jan. 2000.

[15] S. J. Iqbal "Lightning current transfer characteristics of a metal-lattice Tower," M.E. Thesis, Dep. High Voltage Eng., Indian Inst. of Sci., Bangalore, India, Jan. 2003, pp.22.

[16] A. V. Oppenheim, R. W. Schafer, and J. R. Buck, Discrete-Time Signal Processing, 2nd ed., Upper Saddle River, NJ: Prentice Hall, 2000.

[17] Lightning Conductor, in the Book Lightning, R. H. Golde, Ed., vol. 1, New York: Academic, 1977.

[18] IEEE Guide for Improving the Lightning Performance of Transmission Lines, 1997. IEEE Standard, 1243.

Udaya Kumar received the B.E. degree in electrical engineering from Bangalore University, India, in 1989 and both the M.E. and Ph.D. degrees from the Department of High Voltage Engineering, Indian Institute of Science, Bangalore, India, in 1991 and 1997, respectively.

$\mathrm{He}$ is currently an Assistant Professor in the Department of High Voltage Engineering, Indian Institute of Science, Bangalore, India. His major areas of interest are modeling of lightning, analysis and design of lightning protection systems, electromagnetic field analysis pertaining to insulation, and lightning.

Paresh Kumar Nayak received the B.E. degree in electrical engineering from University College of Engineering, Burla, Sambalpur, in 2000 and the M.Sc. degree from the Department of High Voltage of Engineering, Indian Institute of Science, Bangalore, India, in 2003.

His major area of interest is analysis of high-frequency behavior of transformer winding. 